\title{
Existence and uniqueness results for nonlocal integral boundary value problems for fractional differential equations
}

\author{
Suli Liu', Huilai Li ${ }^{1 *}$, Qun Dai ${ }^{2}$ and Junpeng Liu
}

"Correspondence: lihuilai@jlu.edu.cn

${ }^{1}$ School of Mathematics, Jilin

University, Changchun, 130012,

P.R. China

Full list of author information is

available at the end of the article

\begin{abstract}
In this work, we investigate the existence and uniqueness of the solutions for a class of nonlinear fractional differential equations with nonlocal integral boundary conditions. Our analysis relies on the fixed point index theory and a $u_{0}$-positive operator. Examples are discussed for the illustration of the main work.
\end{abstract}

Keywords: fractional differential equations; integral boundary conditions; $u_{0}$-positive operator; fixed point theorem

\section{Introduction}

We consider a class of nonlinear fractional differential equations with nonlocal integral boundary value conditions of this form:

$$
\left\{\begin{array}{l}
D_{0^{+}}^{\alpha} u(t)+p(t) f(t, u(t))=0, \quad 0<t<1, \\
u(0)=u^{\prime}(0)=\cdots=u^{(n-2)}(0)=0 \\
u(1)=\lambda I_{0^{+}}^{\beta} u(\eta)=\lambda \int_{0}^{\eta} \frac{(\eta-s)^{\beta-1} u(s)}{\Gamma(\beta)} \mathrm{d} s
\end{array}\right.
$$

where $\alpha \in(n-1, n]$ is a real number, $n \geq 3$ is an integer, $0<\eta \leq 1, \lambda, \beta>0,0 \leq \frac{\lambda \Gamma(\alpha) \eta^{\alpha+\beta-1}}{\Gamma(\alpha+\beta)}<$ 1 , and $D_{0^{+}}^{\alpha}$ is the standard Riemann-Liouville differential operator.

Here, we emphasize that the integral boundary condition of (1.1) can be understood in the sense that the value of the unknown function at the position $t=1$ is proportional to the Riemann-Liouville fractional integral of the unknown function $\lambda \int_{0}^{\eta} \frac{(\eta-s)^{\beta-1} u(s)}{\Gamma(\beta)} \mathrm{d} s$, where $0<\eta \leq 1$. Furthermore, for $\beta=1$, the integral boundary condition reduces to the usual form of nonlocal integral condition $u(1)=\lambda \int_{0}^{\eta} u(s) \mathrm{d} s$.

Fractional calculus has been investigated in diverse by several researchers. The recent development covers the theoretical as well as potential applications of the subject in physical and technical science. Fractional differential equations have been of great interest recently (see, e.g., [1-5]). There are many results dealing with the existence and multiplicity of solutions of nonlinear fractional differential equations by the means of techniques of nonlinear analysis (see, e.g., [6-12] and the references therein).

(c) 2016 Liu et al. This article is distributed under the terms of the Creative Commons Attribution 4.0 International License (http://creativecommons.org/licenses/by/4.0/), which permits unrestricted use, distribution, and reproduction in any medium, provided you give appropriate credit to the original author(s) and the source, provide a link to the Creative Commons license, and indicate if changes were made. 
Wang and Zhang [13] studied the existence and multiplicity of positive solutions for the following nonlinear fractional differential equations:

$$
\left\{\begin{array}{l}
D_{0^{+}}^{\alpha} u(t)+h(t) f(t, u(t))=0, \quad 0<t<1, n-1<\alpha \leq n, \\
u(0)=u^{\prime}(0)=\cdots=u^{(n-2)}(0)=0, \quad u(1)=\sum_{i=1}^{m-2} \eta_{i} u\left(\xi_{i}\right),
\end{array}\right.
$$

where $\alpha \geq 2, \eta_{i} \geq 0(i=1,2, \ldots, m-2), 0<\xi_{1}<\xi_{2}<\cdots<\xi_{m-2}<1, \sum_{i=1}^{m-2} \eta_{i} \xi_{i}^{\alpha-1}<1, D_{0^{+}}^{\alpha}$ is the standard Riemann-Liouville derivative.

Zhang [14] studied the existence of positive solutions of the following nonlinear fractional differential equation with infinite-point boundary value conditions:

$$
\left\{\begin{array}{l}
D_{0^{+}}^{\alpha} u(t)+q(t) f(t, u(t))=0, \quad 0<t<1 \\
u(0)=u^{\prime}(0)=\cdots=u^{(n-2)}(0)=0 \\
u^{(i)}(1)=\sum_{j=1}^{\infty} \alpha_{j} u\left(\xi_{j}\right)
\end{array}\right.
$$

where $\alpha>2, n-1<\alpha \leq n, i \in[1, n-2]$ is a fixed integer, $\alpha_{j} \geq 0,0<\xi_{1}<\xi_{2}<\cdots<\xi_{j-1}<\xi j<$ $\cdots<1(j=1,2, \ldots), \Delta-\sum_{j=1}^{\infty} \alpha_{j} \xi_{j}^{\alpha-1}>0, \Delta=(\alpha-1)(\alpha-2) \cdots(\alpha-i), D_{0^{+}}^{\alpha}$ is the standard Riemann-Liouville derivative. By introducing height functions of the nonlinear term on some bounded sets and considering integrations of these height functions, several local existence and multiplicity of positive solutions theorems were obtained.

In [15], Ahmad and Agarwal considered the existence and the uniqueness of solutions to a class of Caputo type fractional differential equations of order $q \in(n-1, n]$ with slit-strips type boundary conditions:

$$
\left\{\begin{array}{l}
{ }^{c} D^{q} u(t)=f(t, u(t)), \quad 0<t<1, \\
u(0)=u^{\prime}(0)=\cdots=u^{(n-2)}(0)=0, \\
u(\eta)=a \int_{0}^{\xi} u(s) \mathrm{d} s+b \int_{\zeta}^{1} u(s) \mathrm{d} s
\end{array}\right.
$$

where $0<\xi<\eta<\zeta<1, a$ and $b$ are positive constants.

Liu et al. [16] studied the existence of positive solutions and constructed two successively iterative sequences to approximate the solutions for the following fractional boundary value problem:

$$
\left\{\begin{array}{l}
D_{0^{+}}^{\alpha} u(t)+f(t, u(t))=0, \quad 0<t<1 \\
u(0)=u^{\prime}(0)=u^{\prime \prime}(0)=0 \\
u(1)=\lambda I_{0^{+}}^{\beta} u(\eta)
\end{array}\right.
$$

where $3<\alpha \leq 4,0<\eta \leq 1, \lambda, \beta>0,0 \leq \frac{\lambda \Gamma(\alpha) \eta^{\alpha+\beta-1}}{\Gamma(\alpha+\beta)}<1$, and $D_{0^{+}}^{\alpha}$ is the standard RiemannLiouville differential operator.

For $n=4, p(t) \equiv 1$, the fractional boundary value problem (1.1) reduces to the problem (1.5). For $n=4, \beta=1$, the boundary conditions of (1.1) reduce to $u(0)=u^{\prime}(0)=u^{\prime \prime}(0)=0$, $u(1)=\lambda \int_{0}^{\eta} u(s) \mathrm{d} s$, which had been considered in [17].

Motivated by the work mentioned above, in this article we study the differential equations (1.1) by using $u_{0}$-positive operator and fixed point index theory under some conditions concerning the first eigenvalue with respect to the relevant linear operator. The methods are different from those in previous work; we not only obtain the existence and 
multiplicity of positive solutions for (1.1) under sublinear and superlinear cases, but we also get the uniqueness existence of solutions for (1.1). Moreover, we construct successively iterative sequences to approximate the unique solutions.

This paper is arranged as follows. Some lemmas needed below are listed in Section 2. The existence and multiplicity of the positive solutions to the problem (1.1) are proved in the first part of Section 3. In the second part, one shows the existence and uniqueness of positive solutions and constructs successively iterative sequences to approximate the solutions. Finally, in Section 4, examples are given for the illustration of the main work.

\section{Some lemmas}

Let Banach space $E=C([0,1])$ be endowed with the norm $\|u\|_{\infty}=\max _{0 \leq t \leq 1}|u(t)|$, and $\theta$ is the zero function in $E$. Define a closed cone $P_{c} \subset E$ by $P_{c}=\{u \in E \mid u(t) \geq 0, t \in[0,1]\}$. For any $0<r<R<+\infty$, let $B_{r}=\left\{u \in P_{c}:\|u\|<r\right\}, \partial B_{r}=\left\{u \in P_{c}:\|u\|=r\right\}, B_{R}=\left\{u \in P_{c}:\|u\|<\right.$ $R\}, \bar{B}_{R} \backslash B_{r}=\left\{u \in P_{c}: r \leq\|u\| \leq R\right\}$.

We list the following assumptions adopted in this paper:

$\left(\mathrm{A}_{1}\right) p:[0,1] \rightarrow[0,+\infty)$ is continuous and $0<\int_{0}^{1} p(s) \mathrm{d} s<+\infty$;

$\left(\mathrm{A}_{2}\right) f:[0,1] \times[0, \infty) \rightarrow[0, \infty)$ is continuous and $f(t, 0) \not \equiv$.

For the convenience of the reader, we present here some necessary definitions of the fractional calculus. These definitions can be found in the recent literature [1-3].

Definition 2.1 The Riemann-Liouville fractional integral of order $\alpha>0$ of a function $f$ : $(0, \infty) \rightarrow R$ is given by

$$
I_{0^{+}}^{\alpha} f(t)=\frac{1}{\Gamma(\alpha)} \int_{0}^{t}(t-s)^{\alpha-1} f(s) \mathrm{d} s
$$

provided that the right-hand side is pointwise defined on $(0, \infty)$.

Definition 2.2 The Riemann-Liouville fractional derivative of order $\alpha>0$ of a continuous function $f:(0, \infty) \rightarrow R$ is given by

$$
D_{0^{+}}^{\alpha} f(t)=\frac{1}{\Gamma(n-\alpha)}\left(\frac{d}{d t}\right)^{n} \int_{0}^{t}(t-s)^{n-\alpha-1} f(s) \mathrm{d} s
$$

where $n-1 \leq \alpha<n$, provided that the right-hand side is pointwise defined on $(0, \infty)$.

Lemma 2.1 Assume that $y(t) \in C([0,1])$. The problem

$$
\left\{\begin{array}{l}
D_{0^{+}}^{\alpha} u(t)+y(t)=0, \quad 0<t<1, \\
u(0)=u^{\prime}(0)=\cdots=u^{(n-2)}(0)=0, \\
u(1)=\lambda I_{0+}^{\beta} u(\eta)=\lambda \int_{0}^{\eta} \frac{(\eta-s)^{\beta-1} u(s)}{\Gamma(\beta)} \mathrm{d} s
\end{array}\right.
$$

where $\alpha \in(n-1, n], n \geq 3, n \in N, 0<\eta \leq 1, \lambda, \beta>0,0 \leq \frac{\lambda \Gamma(\alpha) \eta^{\alpha+\beta-1}}{\Gamma(\alpha+\beta)}<1$, is equivalent to

$$
u(t)=\int_{0}^{1} G(t, s) y(s) \mathrm{d} s
$$


where

$$
G(t, s)= \begin{cases}\frac{-P \Gamma(\alpha+\beta)(t-s)^{\alpha-1}+\Gamma(\alpha+\beta)(1-s)^{\alpha-1} t^{\alpha-1}-\Gamma(\alpha) \lambda(\eta-s)^{\alpha+\beta-1} t^{\alpha-1}}{P \Gamma(\alpha) \Gamma(\alpha+\beta)}, & 0 \leq s \leq t \leq 1, s \leq \eta, \\ \frac{\Gamma(\alpha+\beta)(1-s)^{\alpha-1} t^{\alpha-1}-\Gamma(\alpha) \lambda(\eta-s)^{\alpha+\beta-1} t^{\alpha-1}}{P \Gamma(\alpha) \Gamma(\alpha+\beta)}, & 0 \leq t \leq s \leq \eta \leq 1, \\ \frac{-P \Gamma(\alpha+\beta)(t-s)^{\alpha-1}+\Gamma(\alpha+\beta)(1-s)^{\alpha-1} t^{\alpha-1}}{P \Gamma(\alpha) \Gamma(\alpha+\beta)}, & 0 \leq \eta \leq s \leq t \leq 1, \\ \frac{\Gamma(\alpha+\beta)(1-s)^{\alpha-1} t^{\alpha-1}}{P \Gamma(\alpha) \Gamma(\alpha+\beta)}, & 0 \leq t \leq s \leq 1, s \geq \eta,\end{cases}
$$

with $P=1-\frac{\lambda \Gamma(\alpha)}{\Gamma(\alpha+\beta)} \eta^{\alpha+\beta-1}, 0<P \leq 1 . G(t, s)$ is called the Green's function of boundary value problem (2.1). Obviously, $G(t, s)$ is a continuous function on $[0,1] \times[0,1]$.

Proof A function $u \in C^{n-1}[0,1] \cap C^{n}(0,1)$ is called a solution of FBVP (2.1) if it satisfies (2.1). It is shown in [1-3] that problem (2.1) is equivalent to the following integral equation:

$$
u(t)=-I_{0^{+}}^{\alpha} y(t)+C_{1} t^{\alpha-1}+C_{2} t^{\alpha-2}+\cdots+C_{n} t^{\alpha-n}
$$

By $u(0)=u^{\prime}(0)=\cdots=u^{(n-2)}(0)=0$, we have

$$
C_{2}=C_{3}=\cdots=C_{n}=0
$$

Then we get

$$
u(t)=-I_{0^{+}}^{\alpha} y(t)+C_{1} t^{\alpha-1} .
$$

By $u(1)=\lambda I_{0^{+}}^{\beta} u(\eta)$, we have

$$
-I_{0^{+}}^{\alpha} y(1)+C_{1}=-\lambda I_{0^{+}}^{\alpha+\beta} y(\eta)+\lambda C_{1} \frac{\Gamma(\alpha)}{\Gamma(\alpha+\beta)} \eta^{\alpha+\beta-1} .
$$

When $1-\frac{\lambda \Gamma(\alpha)}{\Gamma(\alpha+\beta)} \eta^{\alpha+\beta-1} \neq 0$, we obtain

$$
C_{1}=\frac{1}{1-\frac{\lambda \Gamma(\alpha)}{\Gamma(\alpha+\beta)} \eta^{\alpha+\beta-1}}\left(I_{0^{+}}^{\alpha} y(1)-\lambda I_{0^{+}}^{\alpha+\beta} y(\eta)\right)=: \frac{1}{P}\left(I_{0^{+}}^{\alpha} y(1)-\lambda I_{0^{+}}^{\alpha+\beta} y(\eta)\right) .
$$

Therefore, the solution to problem (2.1) is

$$
\begin{aligned}
u(t)= & -\frac{1}{\Gamma(\alpha)} \int_{0}^{t}(t-s)^{\alpha-1} y(s) \mathrm{d} s+\frac{t^{\alpha-1}}{P \Gamma(\alpha)} \int_{0}^{1}(1-s)^{\alpha-1} y(s) \mathrm{d} s \\
& -\frac{\lambda t^{\alpha-1}}{P \Gamma(\alpha+\beta)} \int_{0}^{\eta}(\eta-s)^{\alpha+\beta-1} y(s) \mathrm{d} s .
\end{aligned}
$$

For $t \leq \eta$, one has

$$
\begin{aligned}
u(t)= & -\frac{1}{\Gamma(\alpha)} \int_{0}^{t}(t-s)^{\alpha-1} y(s) \mathrm{d} s+\frac{t^{\alpha-1}}{P \Gamma(\alpha)}\left\{\int_{0}^{t}+\int_{t}^{\eta}+\int_{\eta}^{1}\right\}(1-s)^{\alpha-1} y(s) \mathrm{d} s \\
& -\frac{\lambda t^{\alpha-1}}{P \Gamma(\alpha+\beta)}\left\{\int_{0}^{t}+\int_{t}^{\eta}\right\}(\eta-s)^{\alpha+\beta-1} y(s) \mathrm{d} s \\
= & \int_{0}^{t} \frac{-P \Gamma(\alpha+\beta)(t-s)^{\alpha-1}+\Gamma(\alpha+\beta)(1-s)^{\alpha-1} t^{\alpha-1}-\Gamma(\alpha) \lambda(\eta-s)^{\alpha+\beta-1} t^{\alpha-1}}{P \Gamma(\alpha) \Gamma(\alpha+\beta)} y(s) \mathrm{d} s
\end{aligned}
$$




$$
\begin{aligned}
& +\int_{t}^{\eta} \frac{\Gamma(\alpha+\beta)(1-s)^{\alpha-1} t^{\alpha-1}-\Gamma(\alpha) \lambda(\eta-s)^{\alpha+\beta-1} t^{\alpha-1}}{P \Gamma(\alpha) \Gamma(\alpha+\beta)} y(s) \mathrm{d} s \\
& +\int_{\eta}^{1} \frac{\Gamma(\alpha+\beta)(1-s)^{\alpha-1} t^{\alpha-1}}{P \Gamma(\alpha) \Gamma(\alpha+\beta)} y(s) \mathrm{d} s \\
& =\int_{0}^{1} G(t, s) y(s) \mathrm{d} s .
\end{aligned}
$$

For $t \geq \eta$, one has

$$
\begin{aligned}
u(t)= & -\frac{1}{\Gamma(\alpha)}\left\{\int_{0}^{\eta}+\int_{\eta}^{t}\right\}(t-s)^{\alpha-1} y(s) \mathrm{d} s+\frac{t^{\alpha-1}}{P \Gamma(\alpha)}\left\{\int_{0}^{\eta}+\int_{\eta}^{t}+\int_{t}^{1}\right\}(1-s)^{\alpha-1} y(s) \mathrm{d} s \\
& -\frac{\lambda t^{\alpha-1}}{P \Gamma(\alpha+\beta)} \int_{0}^{\eta}(\eta-s)^{\alpha+\beta-1} y(s) \mathrm{d} s \\
= & \int_{0}^{\eta} \frac{-P \Gamma(\alpha+\beta)(t-s)^{\alpha-1}+\Gamma(\alpha+\beta)(1-s)^{\alpha-1} t^{\alpha-1}-\Gamma(\alpha) \lambda(\eta-s)^{\alpha+\beta-1} t^{\alpha-1}}{P \Gamma(\alpha) \Gamma(\alpha+\beta)} y(s) \mathrm{d} s \\
& +\int_{\eta}^{t} \frac{-P \Gamma(\alpha+\beta)(t-s)^{\alpha-1}+\Gamma(\alpha+\beta)(1-s)^{\alpha-1} t^{\alpha-1}}{P \Gamma(\alpha) \Gamma(\alpha+\beta)} y(s) \mathrm{d} s \\
& +\int_{t}^{1} \frac{\Gamma(\alpha+\beta)(1-s)^{\alpha-1} t^{\alpha-1}}{P \Gamma(\alpha) \Gamma(\alpha+\beta)} y(s) \mathrm{d} s \\
= & \int_{0}^{1} G(t, s) y(s) \mathrm{d} s .
\end{aligned}
$$

The proof is finished.

Lemma 2.2 ([16]) The Green's function $G(t, s)$ has the following properties:

(1) $G(t, s)>0, \forall t, s \in(0,1)$;

(2) $G(t, s) \leq \frac{(1-s)^{\alpha-1} t^{\alpha-1}}{P \Gamma(\alpha)}, \forall t, s \in(0,1)$;

(3) $G(t, s) \geq \frac{\lambda t^{\alpha-1} \eta^{\alpha+\beta-1}}{P \Gamma(\alpha+\beta)}\left\{(1-s)^{\alpha-1}-(1-s)^{\alpha+\beta-1}\right\}, \forall t, s \in(0,1)$;

(4) $t^{\alpha-1} w_{1}(s) \leq G(t, s) \leq t^{\alpha-1} w_{2}(s), \forall t, s \in(0,1)$,

with $w_{1}(s)=\frac{\lambda \eta^{\alpha+\beta-1}}{P \Gamma(\alpha+\beta)}\left\{(1-s)^{\alpha-1}-(1-s)^{\alpha+\beta-1}\right\}, w_{2}(s)=\frac{(1-s)^{\alpha-1}}{P \Gamma(\alpha)}$.

Definition 2.3 ([18]) We say that a bounded linear operator $T: E \rightarrow E$ is $u_{0}$-positive on the cone $P_{c}$ if there exists $u_{0} \in P_{c} \backslash\{\theta\}$ such that for every $x \in P_{c} \backslash\{\theta\}$ there exist a natural number $n$ and positive functions $\alpha(x)>0, \beta(x)>0$ such that

$$
\alpha(x) u_{0} \leq T^{n} x \leq \beta(x) u_{0}
$$

where $\theta$ is the zero function in $E$. Furthermore, if $u_{0}=\varphi_{1}$, the positive eigenfunction of $T$ corresponding to its first eigenvalue $\lambda_{1}$, then $T$ is a $\varphi_{1}$-positive operator.

Lemma 2.3 (Krein-Rutmann theorem [19]) Suppose that $T: E \rightarrow E$ is a completely continuous linear operator and $T\left(P_{c}\right) \subseteq P_{c}$. If there exist $\psi \in C[0,1] \backslash\left(-P_{c}\right)$ and a constant $c>0$ such that $c T \psi \geq \psi$, then the spectral radius $r(T) \neq 0$ and $A$ has a positive eigenfunction $\varphi_{1}$ corresponding to its first eigenvalue $\lambda_{1}=(r(T))^{-1}$. 
Define the operator $A: C[0,1] \rightarrow C[0,1]$ by

$$
(A u)(t)=\int_{0}^{1} G(t, s) p(s) f(s, u(s)) \mathrm{d} s, \quad t \in[0,1] .
$$

It is not hard to see that the fixed points of operator $A$ coincide with the solutions to the problem (1.1). It is obvious that $A\left(P_{c}\right) \subseteq P_{c}$ when the assumptions $\left(\mathrm{A}_{1}\right)$ and $\left(\mathrm{A}_{2}\right)$ hold. Applying the Arzel-Ascoli theorem, we conclude that $A$ is a completely continuous operator.

Define the operator $T: C[0,1] \rightarrow C[0,1]$ by

$$
(T u)(t)=\int_{0}^{1} G(t, s) p(s) u(s) \mathrm{d} s, \quad t \in[0,1]
$$

It is not difficult to verify that $T: P_{c} \rightarrow P_{c}$ is a completely continuous linear operator. By virtue of the Krein-Rutman theorem, we have the following lemma.

Lemma 2.4 Suppose $T$ is defined by (2.3), then the spectral radius $r(T) \neq 0$ and $A$ has $a$ positive eigenfunction $\varphi_{1}$ corresponding to its first eigenvalue $\lambda_{1}=(r(T))^{-1}$.

Proof By Lemma 2.2, $G(t, s)>0$ for all $t, s \in(0,1)$. Take $\left[t_{1}, t_{2}\right] \subset(0,1), p(t)>0, \forall t \in\left[t_{1}, t_{2}\right]$, choose $\psi \in E$ such that $\psi(t) \geq 0$ for all $t \in[0,1], \psi(t)>0$ for all $t \in\left[t_{1}, t_{2}\right], \psi(t)=0$ for all $t \in\left[0, t_{1}\right) \cup\left(t_{2}, 1\right]$. Thus, we have

$$
(T \psi)(t)=\int_{0}^{1} G(t, s) p(s) \psi(s) \mathrm{d} s=\int_{t_{1}}^{t_{2}} G(t, s) p(s) \psi(s) \mathrm{d} s>0 .
$$

So, there exists a constant $c>0$ such that $c(T \psi)(t) \geq \psi(t), \forall t \in[0,1]$. By Lemma 2.3, we complete the proof.

Lemma 2.5 $T$ is a $u_{0}$-positive operator with $u_{0}(t)=t^{\alpha-1}$. In addition, $T$ is a $\varphi_{1}$-positive operator, where $\varphi_{1}$ is the positive eigenfunction corresponding to its first eigenvalue.

Proof For any $x \in P_{c} \backslash\{\theta\}$, by Lemma 2.2, we have

$$
\begin{aligned}
(T x)(t) & =\int_{0}^{1} G(t, s) p(s) x(s) \mathrm{d} s \\
& \leq \int_{0}^{1} w_{2}(s) p(s) x(s) \mathrm{d} s \cdot t^{\alpha-1} .
\end{aligned}
$$

On the other hand, we have

$$
\begin{aligned}
(T x)(t) & =\int_{0}^{1} G(t, s) p(s) x(s) \mathrm{d} s \\
& \geq \int_{0}^{1} w_{1}(s) p(s) x(s) \mathrm{d} s \cdot t^{\alpha-1} .
\end{aligned}
$$

Therefore, $T$ is a $u_{0}$-positive operator with $u_{0}(t)=t^{\alpha-1}$, i.e.

$$
\alpha(x) u_{0} \leq T x \leq \beta(x) u_{0}, \quad \forall x \in P_{c} \backslash\{\theta\} .
$$


Let $\varphi_{1}$ is the positive eigenfunction of $T$ corresponding to $\lambda_{1}$, i.e. $\varphi_{1}=\lambda_{1} T \varphi_{1}$. Then there exist $\tilde{\alpha}\left(\varphi_{1}\right), \tilde{\beta}\left(\varphi_{1}\right)>0$ such that

$$
\tilde{\alpha}\left(\varphi_{1}\right) u_{0} \leq T \varphi_{1}=\frac{1}{\lambda_{1}} \varphi_{1} \leq \tilde{\beta}\left(\varphi_{1}\right) u_{0}
$$

Hence, we see that $T$ is a $\varphi_{1}$-positive operator.

The proof is completed.

Lemma 2.6 ([18,19]) Let $\Omega \subset E$ be a bounded open set, and $A: \bar{\Omega} \cap P_{c} \rightarrow P_{c}$ is completely continuous.

(i) If there exists $u_{0} \in P_{c} \backslash\{\theta\}$ such that $u-A u \neq \mu u_{0}, \forall \mu \geq 0, u \in \partial \Omega \cap P_{c}$, then $i\left(A, \Omega \cap P_{c}, P_{c}\right)=0$.

(ii) Let $\theta \in \Omega \subset E$, if $u \neq \mu A u, \forall u \in \partial \Omega \cap P_{c}, 0 \leq \mu \leq 1$, then $i\left(A, \Omega \cap P_{c}, P_{c}\right)=1$.

Lemma $2.7([18,19])$ Let $\Omega_{1}$ and $\Omega_{2}$ be two bounded open sets in $E$ such that $\theta \in \Omega_{1}$ and $\bar{\Omega}_{1} \subset \Omega_{2}$. Let operator $A:\left(\bar{\Omega}_{2} \backslash \Omega_{1}\right) \cap P_{c} \rightarrow P_{c}$ is completely continuous. Suppose that one of the two conditions is satisfied:

(i) $A u \ngtr u, \forall u \in P_{c} \cap \partial \Omega_{1}, A u \nless u, \forall u \in P_{c} \cap \partial \Omega_{2}$;

(ii) $A u \nless u, \forall u \in P_{c} \cap \partial \Omega_{1}, A u \ngtr u, \forall u \in P_{c} \cap \partial \Omega_{2}$.

Then $A$ has at least one fixed point in $\left(\Omega_{2} \backslash \bar{\Omega}_{1}\right) \cap P_{c}$.

\section{Main results}

\subsection{Existence and multiplicity results}

For convenience, we list the following assumptions:

$\left(\mathrm{H}_{1}\right) \varliminf_{u \rightarrow 0^{+}} \min _{t \in[0,1]} \frac{f(t, u)}{u}>\lambda_{1}$;

$\left(\mathrm{H}_{2}\right) \varlimsup_{\lim _{u \rightarrow \infty}} \max _{t \in[0,1]} \frac{f(t, u)}{u}<\lambda_{1}$;

$\left(\mathrm{H}_{3}\right) \varlimsup_{u \rightarrow 0^{+}} \max _{t \in[0,1]} \frac{f(t, u)}{u}<\lambda_{1}$;

$\left(\mathrm{H}_{4}\right) \underline{\lim }_{u \rightarrow \infty} \min _{t \in[0,1]} \frac{f(t, u)}{u}>\lambda_{1}$;

$\left(\mathrm{H}_{5}\right)$ there exist $r_{0}>0$, such that

$$
f(t, u(t))<\left[\int_{0}^{1} w_{2}(s) p(s) \mathrm{d} s\right]^{-1} r_{0}, \quad \forall 0<u \leq r_{0}, t \in[0,1]
$$

$\left(\mathrm{H}_{6}\right)$ there exist $\bar{r}_{0}>0$, such that

$$
\begin{gathered}
f(t, u(t))>\left[\int_{\tau}^{1-\tau} \tau^{\alpha-1} w_{1}(s) p(s) \mathrm{d} s\right]^{-1} \bar{r}_{0}, \\
\forall 0<u \leq \bar{r}_{0}, t \in[\tau, 1-\tau] \text {, where } \tau \in(0,1) \text {, such that } p(t) \not \equiv 0, t \in[\tau, 1-\tau] .
\end{gathered}
$$

Theorem 3.1 Suppose that conditions $\left(\mathrm{H}_{1}\right)-\left(\mathrm{H}_{2}\right)$ hold, then the FBVP (1.1) has at least one positive solution.

Proof By $\left(\mathrm{H}_{1}\right)$, there exist $r>0, \varepsilon>0$ such that

$$
f(t, u) \geq\left(\lambda_{1}+\varepsilon\right) u, \quad t \in[0,1], u \in[0, r]
$$


Let $\varphi_{1}$ be the positive eigenfunction of $T$ corresponding to $\lambda_{1}$, i.e. $\varphi_{1}=\lambda_{1} T \varphi_{1}$. For all $u \in \bar{B}_{r} \cap P_{c}$, we have

$$
(A u)(t) \geq\left(\lambda_{1}+\varepsilon\right) \int_{0}^{1} G(t, s) p(s) u(s) \mathrm{d} s=\left(\lambda_{1}+\varepsilon\right)(T u)(t), \quad t \in[0,1] .
$$

Without loss of generality, we may assume that $A$ has no fixed points on $\partial B_{r} \cap P_{c}$. Now we claim that

$$
u-A u \neq \mu \varphi_{1}, \quad \forall u \in \partial B_{r} \cap P_{c}, \mu \geq 0 .
$$

Otherwise, there exist $u_{1} \in \partial B_{r} \cap P_{c}$ and $\mu_{1} \geq 0$ such that $u_{1}-A u_{1}=\mu_{1} \varphi_{1}$, then $u_{1} \geq \mu_{1} \varphi_{1}$. Put $\tau^{*}=\sup \left\{\tau \mid u_{1} \geq \tau \varphi_{1}\right\} . T$ is a positively linear operator, then we have

$$
\left(\lambda_{1}+\varepsilon\right) T\left(u_{1}\right) \geq \lambda_{1} T\left(u_{1}\right) \geq \tau^{*} \lambda_{1} T\left(\varphi_{1}\right)=\tau^{*} \varphi_{1} .
$$

Thus, $u_{1}=A u_{1}+\mu_{1} \varphi_{1} \geq\left(\lambda_{1}+\varepsilon\right) T u_{1}+\mu_{1} \varphi_{1} \geq\left(\tau^{*}+\mu_{1}\right) \varphi_{1}$, which contradicts the definition of $\tau^{*}$. Hence, (3.1) holds. By Lemma 2.6 yields

$$
i\left(A, B_{r} \cap P_{c}, P_{c}\right)=0 .
$$

In addition, by $\left(\mathrm{H}_{2}\right)$, there exist $\varepsilon \in\left(0, \lambda_{1}\right), m>0$ such that $f(t, u) \leq\left(\lambda_{1}-\varepsilon\right) u+m, \forall u \geq R_{1}$, $t \in[0,1]$. Let

$$
\begin{aligned}
& W:=\left\{u \in P_{c} \mid u=\mu A u, \mu \in[0,1]\right\} \\
& \begin{aligned}
u(t) & =\mu(A u)(t) \leq \int_{0}^{1} G(t, s) p(s) f(s, u(s)) \mathrm{d} s \\
& \leq \int_{0}^{1} G(t, s) p(s)\left(\left(\lambda_{1}-\varepsilon\right) u(s)+m\right) \mathrm{d} s \\
& =\left(\lambda_{1}-\varepsilon\right)(T u)(t)+u_{0},
\end{aligned}
\end{aligned}
$$

where $u_{0}:=m \int_{0}^{1} G(t, s) q(s) \mathrm{d} s$. Notice that $r\left(\left(\lambda_{1}-\varepsilon\right) T\right)<1$, thus the operator $I-\left(\lambda_{1}-\varepsilon\right) T$ is invertible. Combining this with (3.4) yields $u \leq\left(\left(\lambda_{1}-\varepsilon\right) T\right)^{-1} u_{0}$, this implies that $W$ is bounded. Choose $R>\max \left\{R_{1}\right.$, sup $\left.W\right\}$, we have

$$
u \neq \mu A u, \quad \forall u \in \partial B_{R} \cap P_{c}, \mu \in[0,1] .
$$

By Lemma 2.6,

$$
i\left(A, B_{R} \cap P_{c}, P_{c}\right)=1 .
$$

By (3.2) and (3.5), we have $i\left(A,\left(B_{R} \backslash \bar{B}_{r}\right) \cap P_{c}, P_{c}\right)=i\left(A, B_{R} \cap P_{c}, P_{c}\right)-i\left(A, B_{r} \cap P_{c}, P_{c}\right)=1$. Hence the operator $A$ has at least one fixed point on $\left(B_{R} \backslash \bar{B}_{r}\right) \cap P_{c}$.

The proof is finished.

Theorem 3.2 Suppose that conditions $\left(\mathrm{H}_{3}\right),\left(\mathrm{H}_{4}\right)$ hold, then the FBVP (1.1) has at least one positive solution. 
Proof The proof is similar to Theorem 3.2 of [13, 17], so we omit the details.

By $\left(\mathrm{H}_{3}\right)$ and Lemma 2.7, we get

$$
i\left(A, B_{r} \cap P_{c}, P_{c}\right)=1 .
$$

By $\left(\mathrm{H}_{4}\right)$ and Lemma 2.7, we get

$$
i\left(A, B_{R} \cap P_{c}, P_{c}\right)=0 .
$$

Hence the operator $A$ has at least one fixed point on $\left(B_{R} \backslash \bar{B}_{r}\right) \cap P_{c}$.

Theorem 3.3 Suppose that conditions $\left(\mathrm{H}_{1}\right),\left(\mathrm{H}_{5}\right)$ hold, then the FBVP (1.1) has at least one positive solution.

Proof Similar to the proof of Theorem 3.1, by $\left(\mathrm{H}_{1}\right)$, we have

$$
i\left(A, B_{r} \cap P_{c}, P_{c}\right)=0 .
$$

By $\left(\mathrm{H}_{5}\right)$, choose $r_{0}>r$, we have

$$
f(t, u(t))<\left[\int_{0}^{1} w_{2}(s) p(s) \mathrm{d} s\right]^{-1} r_{0}, \quad \forall 0<u \leq r_{0}, t \in[0,1] .
$$

Now we claim that

$$
u \neq \mu A u, \quad \forall u \in \partial B_{r_{0}} \cap P_{c}, \mu \in[0,1] .
$$

If otherwise, there exist $u_{1} \in \partial B_{r_{0}} \cap P_{c}$, and $\mu_{1} \in[0,1]$ such that $u_{1}=\mu_{1} A u_{1}$. Noticing

$$
A u_{1}=\int_{0}^{1} G(t, s) p(s) f\left(s, u_{1}(s)\right) \mathrm{d} s<\int_{0}^{1} w_{2}(s) p(s) f\left(s, u_{1}(s)\right) \mathrm{d} s<r_{0}=\left\|u_{1}\right\|, \quad t \in[0,1] .
$$

Thus,

$$
\left\|u_{1}\right\|>\left\|A u_{1}\right\| \geq \mu_{1}\left\|A u_{1}\right\|
$$

which contradicts the fact of $u_{1}=\mu_{1} A u_{1}$. Then (3.9) holds. By Lemma 2.6,

$$
i\left(A, B_{r_{0}} \cap P_{c}, P_{c}\right)=1 .
$$

Therefore, $i\left(A,\left(B_{r_{0}} \backslash \bar{B}_{r}\right) \cap P_{c}, P_{c}\right)=i\left(A, B_{r_{0}} \cap P_{c}, P_{c}\right)-i\left(A, B_{r} \cap P_{c}, P_{c}\right)=1$, then the FBVP (1.1) has at least one positive solution in $\left(B_{r_{0}} \backslash \bar{B}_{r}\right) \cap P_{c}$.

The proof is finished.

Theorem 3.4 Suppose that conditions $\left(\mathrm{H}_{2}\right),\left(\mathrm{H}_{6}\right)$ hold, then the FBVP $(1.1)$ has at least one positive solution. 
Proof By $\left(\mathrm{H}_{2}\right)$, we have

$$
i\left(T, B_{R} \cap P_{c}, P_{c}\right)=1 .
$$

By $\left(\mathrm{H}_{6}\right)$, choose $R>\bar{r}_{0}$, we have

$$
f(t, u(t))>\left[\int_{\tau}^{1-\tau} \tau^{\alpha-1} w_{1}(s) p(s) \mathrm{d} s\right]^{-1} \bar{r}_{0}
$$

$\forall 0<u \leq \bar{r}_{0}, t \in[\tau, 1-\tau]$, where $\tau \in(0,1)$, such that $p(t) \not \equiv 0, t \in[\tau, 1-\tau]$. Now we claim that

$$
A u \nless u, \quad \forall u \in \partial B_{\bar{r}_{0}} \cap P_{c} .
$$

If otherwise, there exist $u_{1} \in \partial B_{\bar{r}_{0}} \cap P_{c}$ such that $A u_{1} \leq u_{1}$. Noticing

$$
\begin{aligned}
A u_{1} & =\int_{0}^{1} G(t, s) p(s) f\left(s, u_{1}(s)\right) \mathrm{d} s \geq \int_{0}^{1} s^{\alpha-1} w_{1}(s) p(s) f\left(s, u_{1}(s)\right) \mathrm{d} s \\
& >\int_{\tau}^{1-\tau} \tau^{\alpha-1} w_{1}(s) p(s) f\left(s, u_{1}(s)\right) \mathrm{d} s>\bar{r}_{0}=\left\|u_{1}\right\|, \quad t \in[0,1] .
\end{aligned}
$$

Thus, $\left\|A u_{1}\right\|>\left\|u_{1}\right\|$, which contradicts the fact of $A u \nless u$. Then (3.12) holds. By Lemma 2.7,

$$
i\left(A, B_{\bar{r}_{0}} \cap P_{c}, P_{c}\right)=0 .
$$

Thus the FBVP (1.1) has at least one positive solution in $\left(B_{R} \backslash \bar{B}_{\bar{r}_{0}}\right) \cap P_{c}$.

The proof is finished.

Similarly, we can get the following result.

Corollary 3.1 Suppose that conditions $\left(\mathrm{H}_{4}\right),\left(\mathrm{H}_{5}\right)$ hold, then the FBVP (1.1) has at least one positive solution.

Corollary 3.2 Suppose that conditions $\left(\mathrm{H}_{3}\right),\left(\mathrm{H}_{6}\right)$ hold, then the FBVP (1.1) has at least one positive solution.

Corollary 3.3 Suppose that conditions $\left(\mathrm{H}_{1}\right),\left(\mathrm{H}_{4}\right),\left(\mathrm{H}_{5}\right)$ hold, then the FBVP (1.1) has at least two positive solutions.

Corollary 3.4 Suppose that conditions $\left(\mathrm{H}_{2}\right),\left(\mathrm{H}_{3}\right),\left(\mathrm{H}_{6}\right)$ hold, then the FBVP (1.1) has at least two positive solutions.

\subsection{Uniqueness results}

Theorem 3.5 Suppose that there exists $k \in[0,1)$ such that

$$
|f(t, u)-f(t, v)| \leq k \lambda_{1}|u-v|, \quad \forall t \in[0,1], u, v \in P_{c}
$$


where $\lambda_{1}$ is the first eigenvalue of T. Then the FBVP (1.1) has a unique positive solution $u^{*}$, moreover, for any $u_{0} \in P_{c}$, there exist iterative sequences $\left\{u_{n}\right\}_{n=0}^{\infty}$ with

$$
u_{n+1}=A u_{n}, \quad \lim _{n \rightarrow \infty} u_{n}=u^{*}, n=0,1,2, \ldots
$$

Proof First of all, it is not hard to see that the fixed points of operator $A$ coincide with the solutions to the problem (1.1).

Second, we will show that $A$ has fixed points in $P_{c}$. For any given $u_{0} \in P_{c}$, let $u_{n+1}=A u_{n}$. By Lemma 2.5 , there exists $\beta=\beta\left(\left|u_{1}-u_{0}\right|\right)>0$, such that

$$
\left(T\left|u_{1}-u_{0}\right|\right)(t) \leq \beta \varphi_{1}(t), \quad t \in[0,1]
$$

Then, for any $m \in \mathbb{N}$, we have

$$
\begin{aligned}
\left|u_{m+1}-u_{m}\right| & =\left|\left(A u_{m}\right)(t)-\left(A u_{m-1}\right)(t)\right| \\
& =\left|\int_{0}^{1} G(t, s) p(s)\left[f\left(s, u_{m}(s)\right)-f\left(s, u_{m-1}(s)\right)\right] \mathrm{d} s\right| \\
& \leq \int_{0}^{1} G(t, s) p(s)\left|f\left(s, u_{m}(s)\right)-f\left(s, u_{m-1}(s)\right)\right| \mathrm{d} s \\
& \leq k \lambda_{1} \int_{0}^{1} G(t, s) p(s)\left|u_{m}-u_{m-1}\right| \mathrm{d} s \\
& =k \lambda_{1} T\left(\left|u_{m}-u_{m-1}\right|\right)(t) \leq \cdots \\
& \leq k^{m} \lambda_{1}^{m} T^{m}\left(\left|u_{1}-u_{0}\right|\right)(t) \\
& \leq k^{m} \lambda_{1}^{m} T^{m-1} \beta \varphi_{1}(t)=\beta k^{m} \lambda_{1}^{m-1} T^{m-2} \varphi_{1}(t) \\
& =\beta k^{m} \lambda_{1} \varphi_{1}(t) .
\end{aligned}
$$

Thus, for $n, m \in \mathbb{N}$, we have

$$
\begin{aligned}
\left|u_{n+m+1}-u_{n}\right| & =\left|u_{n+m+1}-u_{n+m}+\cdots+u_{n+1}-u_{n}\right| \\
& \leq\left|u_{n+m+1}-u_{n+m}\right|+\cdots+\left|u_{n+1}-u_{n}\right| \\
& \leq \beta\left[k^{n+m}+\cdots+k^{n}\right] \lambda_{1} \varphi_{1}(t) \\
& =\beta \lambda_{1} \frac{k^{n}\left(1-k^{m+1}\right)}{1-k} \varphi_{1}(t) .
\end{aligned}
$$

Therefore,

$$
0 \leq\left\|u_{n+m+1}-u_{n}\right\| \leq \beta \lambda_{1} \frac{k^{n}\left(1-k^{m+1}\right)}{1-k}\left\|\varphi_{1}(t)\right\| \rightarrow 0, \quad \text { as } n, m \rightarrow 0 .
$$

By the completeness of $E$, there exist a $u^{*} \in P_{c}$ such that $\lim _{n \rightarrow \infty} u_{n}=u^{*}$.

Thus, $u^{*}=\lim _{n \rightarrow \infty} u_{n+1}=\lim _{n \rightarrow \infty} A u_{n}=A u^{*}, A$ have fixed points in $P_{c}$.

Finally, we will show that $A$ has at most one fixed point in $P_{c}$. 
Suppose there exist two fixed points $u, v \in P_{c}, u=A u, v=A v$. By Lemma 2.5, there exists $\beta=\beta(|u-v|)>0$, such that

$$
(T|u-v|)(t) \leq \beta \varphi_{1}(t), \quad t \in[0,1] .
$$

Then $\forall n \in \mathbb{N}$, the following hold:

$$
|u-v|=\left|A^{n} u-A^{n} v\right| \leq \beta k^{m} \lambda_{1} \varphi_{1} .
$$

This means that $u=v, A$ has at most one fixed point in $P_{c}$.

The proof is completed.

Remark 3.1 The iterative sequences in Theorem 3.5 starting with a simple function is helpful for calculating.

By the same method of [12], we have the following results. We omit the details.

Corollary 3.5 Suppose that there exists $u_{0} \in P_{c}$ such that

$$
\begin{aligned}
& D_{0^{+}}^{\alpha} u_{0}(t)+p(t) f\left(t, u_{0}(t)\right) \geq 0, \quad 0<t<1, \\
& u_{0}(0)=u_{0}^{\prime}(0)=\cdots=u_{0}^{(n-2)}(0)=0, \quad u_{0}(1) \leq \lambda I_{0^{+}}^{\beta} u_{0}(\eta),
\end{aligned}
$$

and $\forall u, v \in \Omega, u(t) \geq v(t)$, there exist $k \in[0,1)$ such that

$$
0 \leq f(t, u(t))-f(t, v(t)) \leq k \lambda_{1}(u(t)-v(t))
$$

where $\Omega=\left\{u \in P_{c} \mid u \geq u_{0}\right\}$. Then FBVP (1.1) has a unique solution $u^{*}$ with $\lim _{n \rightarrow \infty} u_{n}=u^{*}$, $u_{n+1}=A u_{n}, n=0,1,2, \ldots$

Corollary 3.6 Suppose that there exists $u_{0} \in P_{c}$ such that

$$
\begin{aligned}
& D_{0^{+}}^{\alpha} u_{0}(t)+p(t) f\left(t, u_{0}(t)\right) \leq 0, \quad 0<t<1, \\
& u_{0}(0)=u_{0}^{\prime}(0)=\cdots=u_{0}^{(n-2)}(0)=0, \quad u_{0}(1) \geq \lambda I_{0^{+}}^{\beta} u_{0}(\eta),
\end{aligned}
$$

and $\forall u, v \in \Omega, u(t) \geq v(t)$, there exist $k \in[0,1)$ such that

$$
0 \leq f(t, u(t))-f(t, v(t)) \leq k \lambda_{1}(u(t)-v(t))
$$

where $\Omega=\left\{u \in P_{c} \mid u \leq u_{0}\right\}$. Then FBVP (1.1) has a unique solution $u^{*}$ with $\lim _{n \rightarrow \infty} u_{n}=u^{*}$, $u_{n+1}=A u_{n}, n=0,1,2, \ldots$.

\section{Examples}

Example 4.1 Consider the following boundary value problem:

$$
\left\{\begin{array}{l}
D_{0+}^{7 / 2} u(t)+\frac{\lambda_{0}(1-t)^{-1 / 2}}{2\left(1+\lambda_{0}\right)}\left[3 u+1+\sin u+u^{2}\right]=0, \quad 0<t<1, \\
u(0)=u^{\prime}(0)=u^{\prime \prime}(0)=0, \quad u(1)=5 I_{0+}^{3 / 2} u\left(\frac{1}{2}\right),
\end{array}\right.
$$


where $\alpha=\frac{7}{2}, \beta=\frac{3}{2}, \eta=\frac{1}{2}, \lambda=5,0 \leq \frac{\lambda \Gamma(\alpha) \eta^{\alpha+\beta-1}}{\Gamma(\alpha+\beta)} \approx 0.0433<1$, and $p(t)=\frac{(1-t)^{-1 / 2}}{1+\lambda_{0}}, f(t, u(t))=$ $\frac{1}{2} \lambda_{0}\left[3 u+1+\sin u+u^{2}\right], \lambda_{0}>\lambda_{1}, \lambda_{1}$ is the first eigenvalue of the operator $T$.

By simple computation, it is clear that $\left(\mathrm{A}_{1}\right),\left(\mathrm{A}_{2}\right),\left(\mathrm{H}_{1}\right),\left(\mathrm{H}_{4}\right)$ hold.

Choose $r_{0}=1$, then $\forall 0<u \leq 1, t \in[0,1]$, we have

$$
f(t, u(t))=\frac{1}{2} \lambda_{0}\left[3 u+1+\sin u+u^{2}\right] \leq 3 \lambda_{0}, \quad\left[\int_{0}^{1} w_{2}(s) p(s) d s\right]^{-1} r_{0} \approx 9.538\left(1+\lambda_{0}\right)
$$

Thus, $\left(\mathrm{H}_{5}\right)$ holds.

It follows from Corollary 3.3 that FBVP (4.1) has at least two positive solutions.

Example 4.2 Consider the following boundary value problem:

$$
\left\{\begin{array}{l}
D_{0+}^{7 / 2} u(t)+\lambda_{0}(1-t)^{-1 / 2}\left[\frac{1}{3} u+2+t^{3}+\sin t+\frac{1}{2} u^{1 / 2}\right]=0, \quad 0<t<1 \\
u(0)=u^{\prime}(0)=u^{\prime \prime}(0)=0, \quad u(1)=5 I_{0+}^{3 / 2} u\left(\frac{1}{2}\right)
\end{array}\right.
$$

where $\alpha=\frac{7}{2}, \beta=\frac{3}{2}, \eta=\frac{1}{2}, \lambda=5,0 \leq \frac{\lambda \Gamma(\alpha) \eta^{\alpha+\beta-1}}{\Gamma(\alpha+\beta)} \approx 0.0433<1$, and $p(t)=(1-t)^{-1 / 2}$, $f(t, u(t))=\lambda_{0}\left[\frac{1}{3} u+2+t^{3}+\sin t+\frac{1}{2} u^{1 / 2}\right], 0<\lambda_{0}<\lambda_{1}, \lambda_{1}$ is the first eigenvalue of the operator $T$.

It is clear that $\left(A_{1}\right),\left(A_{2}\right)$ hold.

For $\forall u, v \in P_{c}$, we have

$$
|f(t, u(t))-f(t, v(t))| \leq \lambda_{0}\left|\left[\frac{1}{3}(u-v)+\frac{1}{2}\left(u^{1 / 2}-v^{1 / 2}\right)\right]\right| \leq \lambda_{1} \frac{5}{6}|u-v|
$$

It follows from Theorem 3.5 that FBVP (4.2) has a unique positive solution, moreover, for any $u_{0} \in P_{c}$, there exist iterative sequences $\left\{u_{n}\right\}_{n=0}^{\infty}$ with

$$
u_{n+1}=A u_{n}, \quad \lim _{n \rightarrow \infty} u_{n}=u^{*}, n=0,1,2, \ldots
$$

\section{Competing interests}

The authors declare that they have no competing interests.

\section{Authors' contributions}

All authors contributed equally to the manuscript. All authors read and approved the final manuscript.

\section{Author details}

'School of Mathematics, Jilin University, Changchun, 130012, P.R. China. ${ }^{2}$ College of Science, Changchun University of Science and Technology, Changchun, 130022, P.R. China.

\section{Acknowledgements}

The authors are grateful to anonymous referees for their constructive comments and suggestions, which have greatly improved this paper. This work is supported by NSF of China (11271154).

Received: 31 October 2015 Accepted: 25 April 2016 Published online: 03 May 2016

\section{References}

1. Samko, SG, Kilbas, AA, Marichev, Ol: Fractional Integrals and Derivatives: Theory and Applications. Gordon \& Breach, Yverdon (1993)

2. Miller, KS, Ross, B: An Introduction to the Fractional Calculus and Fractional Differential Equations. Wiley, New York (1993)

3. Podlubny, I: Fractional Differential Equations: An Introduction to Fractional Derivatives, Fractional Differential Equations, to Methods of Their Solution and Some of Their Applications, vol. 198. Academic Press, San Diego (1998)

4. Kilbas, AAA, Srivastava, HM, Trujillo, JJ: Theory and Applications of Fractional Differential Equations, vol. 204. Elsevier, Amsterdam (2006) 
5. Sabatier, J, Agrawal, OP, Machado, JT: Advances in Fractional Calculus, vol. 4. Springer, Dordrecht (2007)

6. Lakshmikantham, V, Vatsala, AS: Basic theory of fractional differential equations. Nonlinear Anal., Theory Methods Appl. 69(8), 2677-2682 (2008)

7. Benchohra, M, Graef, JR, Hamani, S: Existence results for boundary value problems with non-linear fractional differential equations. Appl. Anal. 87(7), 851-863 (2008)

8. Bai, Z: On positive solutions of a nonlocal fractional boundary value problem. Nonlinear Anal., Theory Methods Appl. 72(2), 916-924 (2010)

9. Xie, W, Xiao, J, Luo, Z: Existence of extremal solutions for nonlinear fractional differential equation with nonlinear boundary conditions. Appl. Math. Lett. 41, 46-51 (2015)

10. Alsaedi, A, Ntouyas, SK, Agarwal, RP, Ahmad, B: On Caputo type sequential fractional differential equations with nonlocal integral boundary conditions. Adv. Differ. Equ. 2015, 33 (2015)

11. Zhai, C, Hao, M: Mixed monotone operator methods for the existence and uniqueness of positive solutions to Riemann-Liouville fractional differential equation boundary value problems. Bound. Value Probl. 2013, 85 (2013). doi:10.1186/1687-2770-2013-85

12. Cui, Y: Uniqueness of solution for boundary value problems for fractional differential equations. Appl. Math. Lett. 51, 48-54 (2016)

13. Wang, $L$, Zhang, $X$ : Positive solutions of $m$-point boundary value problems for a class of nonlinear fractional differential equations. J. Appl. Math. Comput. 42(1-2), 387-399 (2013). doi:10.1007/s12190-012-0626-0

14. Zhang, $X$ : Positive solutions for a class of singular fractional differential equation with infinite-point boundary value conditions. Appl. Math. Lett. 39, 22-27 (2015)

15. Ahmad, B, Agarwal, R: Some new versions of fractional boundary value problems with slit-strips conditions. Bound. Value Probl. 2014, 175 (2014). doi:10.1186/s13661-014-0175-6

16. Liu, S, Li, H, Dai, Q: Nonlinear fractional differential equations with nonlocal integral boundary conditions. Adv. Differ Equ. 2015, 187 (2015)

17. Zhang, X, Wang, L, Sun, Q: Existence of positive solutions for a class of nonlinear fractional differential equations with integral boundary conditions and a parameter. Appl. Math. Comput. 226, 708-718 (2014)

18. Krasnosel'skii, M: Positive Solution of Operator Equation. Noordhoff, Groningen (1964)

19. Guo, D, Sun, J: Nonlinear Integral Equations. Shandong Science and Technology Press, Jinan (1987)

\section{Submit your manuscript to a SpringerOpen ${ }^{\circ}$ journal and benefit from:}

- Convenient online submission

Rigorous peer review

- Immediate publication on acceptance

- Open access: articles freely available online

- High visibility within the field

- Retaining the copyright to your article 